Anaesthesist 2021 - 70:618-620

https://doi.org/10.1007/s00101-021-01006-6

Angenommen: 9. Juni 2021

Online publiziert: 18. Juni 2021

(c) Springer Medizin Verlag $\mathrm{GmbH}$, ein Teil von Springer Nature 2021

\section{Die intensivmedizinische Behandlung der schwangeren Patientin}

\author{
Ein ausführlicher Exkurs in vier Schritten
}

Vor fast 20 Jahren wurde in einem Editorial in der Zeitschrift Der Anaesthesist der Gedanke ausgeführt, dass Analgesien unter der Geburt "Geschichten aus tausendundeiner Nacht von Schritten zurück zum Garten Eden" sind [1]. Ein Gedanke, der aus der üblichen Prosa einer medizinischen Fachzeitschrift sicherlich herausragt. Schwangerschaft und Geburt, das aber ist im Leben jedes Einzelnen meist etwas ganz Besonderes: "Schwangerschaft und Geburt, ein freudiges Ereignis!", so auch die weit verbreitete Wahrnehmung in der Bevölkerung. Oft werden die damit verbundenen Risiken und die dann manchmal notwendige (Intensiv-)Medizin von den Betroffenen und Angehörigen geradezu ausgeblendet. Als Anästhesiolog*in ist die Begleitung von schwangeren Patientinnen im Kreißsaal und auf der Intensivstation nicht immer von alleinig froher Erwartung geprägt - oft ist sie mehr mit der Hoffnung verbunden, dass es wieder mal gut gehen möge - wissend, dass dies keineswegs garantiert ist. Uns Anästhesist*innen und

Die Autoren Thomas Fuchs-Buder, Axel R. Heller, Markus Rehm, Markus Weigand und Alexander Zarbock sind die Herausgeber der Rubrik „CME Zertifizierte Fortbildung" der Zeitschrift. Ein herzlicher Dank der Rubrikherausgeber für ihre Unterstützung geht an Frau Dr. Meidert.
Intensivmediziner*innen ist bewusst, dass Schwangerschaft und Geburt im Leben einer Frau selbst in der westlichen Welt noch immer ein potenziell lebensbedrohliches Ereignis darstellt. Durch die medizinischen Anstrengungen in den letzten Jahrzehnten ist die Müttersterblichkeit in Deutschland mit etwa 7/100.000 Lebendgeborene im weltweiten Vergleich erfreulich niedrig, dennoch ist jede werdende Mutter, die eine Geburt nicht überlebt, eine zu viel.

Gerade durch die umfangreichen Anpassungsvorgänge während der Schwangerschaft sind Anästhesist*innen und Intensivmediziner/-innen gefordert, sich mit den physiologischen, aber auch pathophysiologischen Veränderungen während und nach einer Schwangerschaft detailliert auseinanderzusetzen. Dem wird u. a. dadurch gut Rechnung getragen, dass auf Kongressen und anderen Fortbildungsveranstaltungen den Sitzungen, die Aspekte dieses Themas beinhalten, große Aufmerksamkeit zuteilwird; sie werden meist von besonders vielen Zuhörern besucht. Geburt und das „Danach“ ist natürlich ein Thema, das jeden ganz persönlich betreffen kann. Werdende Mütter sind zudem ganz besondere Patientinnen. Wenn Schwangere keine erheblichen Vorerkrankungen mitbringen, erwarten sie heute meist hoff- nungsvoll „lediglich" ein Kind und nicht, kritisch krank zu werden oder gar zu sterben. Selbst bei Patientinnen mit komplexen Herzvitien kann man häufig beobachten, dass die bestehende, oft sehr schwere Erkrankung mit einer Schwangerschaft nahezu komplett ausgeblendet wird. Es setzt geradezu ein "neglect" ein; Kontrollbefunde werden plötzlich nicht mehr zuverlässig beigebracht, Kontrolltermine abgesagt oder versäumt. Manchmal bedeutet dies eine große Herausforderung für Geburtshelfer und all die anderen Fachdisziplinen, die die werdende Mutter mitbetreuen.

Unzweifelhaft ist also von außerordentlich großer Wichtigkeit, dass das Behandlungsteam auf die spezifischen Krankheitsbilder während der Schwangerschaft und Geburt gut vorbereitet ist, damit die Behandlung selbst in dramatischen Situationen möglichst kompetent erfolgt. Aus diesem Grund haben wir, als Herausgeber der Rubrik CME Zertifizierte Fortbildung, diesem Thema eine Artikelreihe gewidmet und in den folgenden Ausgaben von Der Anaesthesist in 4 Schritten ausgeführt. Für die 4 Artikel konnten wir hochkarätige Autoren gewinnen:Thema des ersten Teils der Reihe sind die grundlegenden Prinzipien und aktuellen Empfehlungen bei der intensivmedizinischen Versorgung schwan- 


\section{Infobox 1}

Dieses Editorial leitet 4 aufeinanderfolgende CME-Beiträge zur schwangeren Patientin in Der Anaesthesist ein. Die 4 Beiträge der Reihe sind unabhängig voneinander, ergänzen sich aber thematisch:

- „Prinzipien zur intensivmedizinischen Versorgung der schwangeren Patientin", www.springermedizin.de/link/10.1007/ s00101-021-00947-2

- „Spezielle intensivmedizinische Krankheitsbilder der schwangeren Patientin", www.springermedizin.de/link/10.1007/ s00101-021-00946-3

- „Internistische Notfälle der schwangeren Patientin", www.springermedizin.de/link/ 10.1007/s00101-021-00944-5

- „Vaskuläre Notfälle bei der schwangeren Patientin", www.springermedizin.de/link/ 10.1007/s00101-021-00945-4

Bitte beachten Sie, dass die Arbeiten nacheinander erscheinen. Sie finden sie auf www.springermedizin.de. Bitte geben Sie dort den Titel in die Suche ein. Der CME-Kurs kann erst mit Erscheinen der jeweiligen Druckausgabe absolviert werden.

gerer und kürzlich entbundener Patientinnen, gefolgt von einem Beitrag zu speziellen intensivmedizinischen Krankheitsbildern in der Schwangerschaft, didaktisch ausgezeichnet aufbereitet durch Neuhaus et al. Die 2 folgenden, ebenso hervorragend gestalteten Beiträge von Fischer et al. befassen sich mit den internistischen Krankheitsbildern in der geburtshilflichen Intensivmedizin (einschließlich der peripartalen Sepsis) und den vaskulären Krankheitsbildern, die auch die Intensivmedizin unmittelbar betreffen.

\section{॥ Die in vielen Aspekten extreme Situation der schwangeren Patientin erfordert hohe medizinische Kompetenz}

Ein umschriebenes und damit auch gut eingrenzbares Themenfeld - möchte man meinen. Warum aber entsteht dann bei Anästhesist*innen und Intensivmedizinern/ Intensivmedizinerinnen trotzdem manchmal eine gewisse Sorge bis hin sogar zum Unbehagen bei der Ankündigung einer schwangeren Patientin, selbst bei Routineprozeduren? Neben der Tatsache, mit Mutter und Ungeborenem immer zwei vulnerable Patienten gleichzeitig zu betreuen, ist für Anästhesiolog*innen die Beglei- tung einer schwangeren Patientin auf der Intensivstation oder während einer Sectio caesarea aus einem weiteren Grund außergewöhnlich: Meist sind die Patientinnen wach, oft ist zudem ein besorgter werdender Vater vor Ort. Es besteht in der Regel nicht die Option, die Situation durch eine leichte Sedierung „erträglicher" zu machen, wie es bei anderen Eingriffen in Spinalanästhesie gebräuchlich ist. Das Paar befindet sich in einer Ausnahme- und Extremsituation, deshalb sind Ärzt*innen nicht nur hinsichtlich der Physis der Patientin, sondern auch hinsichtlich deren mentaler/psychischer Begleitung ganz besonders gefordert. Zu den belastenden, durchaus auch traumatisierenden und manchmal sogar verschwiegenen Faktoren, die ein Paar mitbringen kann, gehören nicht selten ein Abort in der Vorgeschichte oder eine aufreibende Kinderwunschbehandlung. Die enorme Bedeutung guter und beruhigender Kommunikation kann an dieser Stelle gar nicht genug betont werden. Anschaulich zu beobachten ist der außerordentliche Stellenwert der Beruhigung für die Mutter, wenn während Sectio der Säugling schreit und dann quicklebendig zur Mutter gebracht wird: Man kann es förmlich messen, die Herzfrequenz sinkt nach initialem abrupten Anstieg durch die erlebte große Freude, der Blutdruck steigt und bedarf in den meisten Fällen keiner Therapie mehr (trotz stattgehabtem Blutverlust und Regionalverfahren).

Die Geburt ist unzweifelhaft etwas ganz Besonderes im Leben einer Mutter. So wird gut verständlich, dass evtl. damit einhergehende Komplikationen das Leben einer jungen Frau auf lange Zeit hinaus tief beeinflussen können. Eine spanische Erhebung aus dem Jahr 2019 fand heraus, dass allein ein Intensivaufenthalt der Mutter die Lebensqualität noch nach Jahren in etwa genauso stark beeinträchtigt wie ein hochgradiger Dammriss und sogar deutlich stärker als Frühgeburtlichkeit des Kindes [2].

Grundvoraussetzung für eine gute peripartale Betreuung der Mütter ist eine möglichst hohe medizinische Kompetenz. Hier stellen die 4 Fortbildungsartikel eine echte Bereicherung dar, für die allen Autoren an dieser Stelle ganz herzlich gedankt wird. Die Mitherausgeber der Rubrik freuen sich sehr, dass die Autoren ein Gesamtkonzept umsetzten, in dem der aktuelle medizinische Kenntnisstand und der medizinische Fortschritt auf diesem wichtigen Gebiet schrittweise aufbereitet wurden. Die Beiträge behandeln allerdings nicht ausführlich die oben angesprochene mentale peripartale Begleitung. Hier findet sich in der anästhesiologischen Literatur aber auch noch zu wenig. Auf diesem Gebiet gibt es also noch viel zu forschen, zu entwickeln viel Raum also für weitere „Geschichten aus tausendundeiner Nacht von Schritten zurück zum Garten Eden“. Neben dem reinen Überleben der Komplikationen von Schwangerschaft und Geburt liegt es auch an unserem Fachgebiet - und damit an jedem Einzelnen -, die Frauen kompetent in den Beginn einer neuen Lebensphase zu begleiten, sodass die "gute Hoffnung" physisch und psychisch gerechtfertigt sein darf.

\section{Korrespondenzadresse}

Prof. Dr. Markus Rehm

Klinik für Anaesthesiologie, Klinikum der Universität München, Campus Großhadern Marchioninistr. 15, 81377 München, Deutschland

markus.rehm@med.uni-muenchen.de

Interessenkonflikt. A. Meidert, T. Fuchs-Buder, A.R. Heller, M. Weigand, A. Zarbock und M. Rehm geben an, dass kein Interessenkonflikt besteht.

\section{Literatur}

1. Rehm M (2002) Analgesie unter der Geburt Geschichten aus tausendundeiner Nacht von Schritten zurück zum Garten Eden. Anaesthesist 51(12):955. https://doi.org/10.1007/s00101-0020431-5

2. Martínez-Galiano JM, Hernández-Martínez A, Rodríguez-Almagro J, Delgado-Rodríguez $M$ (2019) Quality of Life of Women after Giving Birth: Associated Factors Related with the Birth Process. J Clin Med 8(3):324. https://doi.org/10. 3390/jcm8030324 
Hier steht eine Anzeige.

黑 Springer 